\title{
Effect of aging process on combustion and mechanical performance of nitroguanidine propellants
}

\author{
Peng Wang ${ }^{1, *}$, Zhitao $\mathrm{Liu}^{1}, \mathrm{Hao}_{\mathrm{Guo}}{ }^{2}$, and Xin Liao ${ }^{1}$ \\ ${ }^{1}$ School of Chemical Engineering, Nanjing University of Science and Technology, Nanjing 210094, \\ China \\ ${ }^{2}$ Qing Yang Chemical Industry Corporation, Liaoyang 111000, China
}

\begin{abstract}
Keywords: aging process, combustion and mechanical performance, nitroguanidine propellants.
\end{abstract}

\begin{abstract}
The effect of different aging times (1 day, 3 days, 5 days and 7 days) on the combustion performance and mechanical properties of nitroguanidine propellants was studied.Through the mechanical properties test of impact and compression and closed bomb test of the prepared propellants, it was found that the relative steepness of the propellants aged for 3 days under normal and low temperature conditions was all less than $1 ;$ Under normal and low temperature conditions, the impact strength of propellants aged for 5 days was the largest;Propellants aged for 7 days had the highest compressive strength at low temperatures and propellants aged for 1 day had the highest compression rate at low temperatures.Comprehensive research results show that aging time of three days has a better improvement in the combustion performance and mechanical properties of the propellants.
\end{abstract}

\section{Introduction}

Nitroguanidine propellants are three-base propellants made of double-base propellants plus a certain amount of nitroguanidine, triguanidine for short [1]. Nitroguanidine propellants have high energy and low explosion temperature, which meet the development requirements of modern weapons [2].However, nitroguanidine propellants are a kind of heterogeneous and high solid content gunpowder and its mechanical properties are not good [3]. In addition, the mechanical properties of nitroguanidine propellants at low temperature are not very good [4].Specifically, abnormal combustion sometimes occurs at low temperatures, which brings safety hazards [5].Therefore, it is of great significance to improve the mechanical properties and combustion performance of nitroguanidine propellants.

At present, the domestic research on nitroguanidine propellants mainly focuses on the effect of nitrocellulose types, alcohol-to-ketone ratio, plasticizing temperature and time, compression and stretching methods on its performance [6].However, there are few studies

*Corresponding author: 243614959@.qq.com 
on the effect of aging time (the time for bolus to be kept tightly closed from after plasticization to before compression and stretching) on its performance.Aging can relax and restore the spatial configuration of $\mathrm{NC}$ fibers in the bolus and improve the internal micromechanical structure of the propellants [7].Thus reducing stress concentration and other problems in the preparation process of propellant and improving the mechanical properties and combustion performance [8].

This article mainly studies the effect of aging time on the combustion performance and mechanical properties of nitroguanidine propellants, so as to obtain the best preparation process of nitroguanidine propellants.

\section{Reagents and Instruments}

Reagents: Rod Nitroguanidine, Liaoning Qingyang Special Chemical Co., Ltd.;

Double base absorption tablets,Liaoning Qingyang Special Chemical Co., Ltd.;

Potassium sulfate, chemically pure, Sinopharm Chemical Reagent Co.,Ltd;

Anhydrous ethanol, chemically pure, Nanjing Chemical Reagent Co., Ltd;

Acetone, chemically pure, Nanjing Chemical Reagent Co., Ltd.

Instruments: Instron3367 universal material testing machine, Instron Corporation.

\section{Experiment procedure}

\subsection{Preparation of nitroguanidine propellants.}

The nitroguanidine propellants are prepared by semi-solvent method.The aging process is as follows: The gelled materials should be placed in a closed environment at a temperature of $20^{\circ} \mathrm{C}$ for a period of time. Then, press and stretch forming and the following process are carried out, as shown in Fig. 1. Taking nitroguanidine propellants as the research object, the gelled materials are aged for $0,1,3,5$, and 7 days and then prepared into $15 / 1$ propellant samples for research.

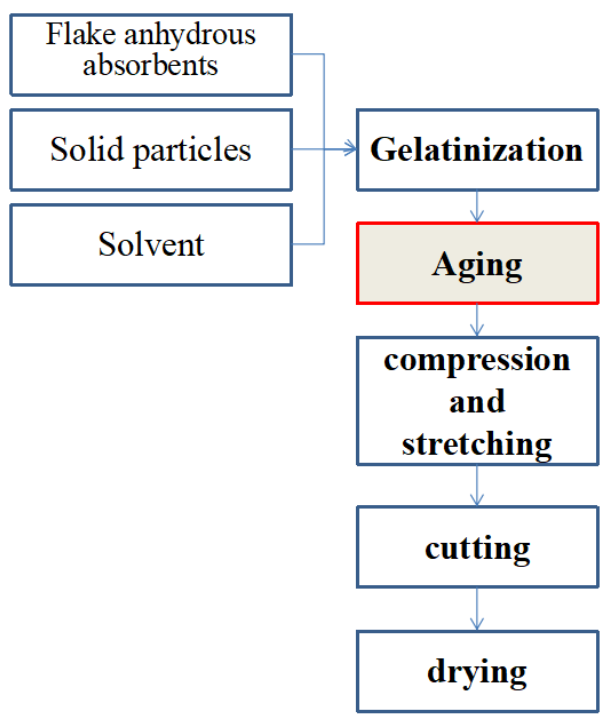

Fig. 1. Process flow of propellant preparation through aging and semi-solvent method. 


\subsection{Mechanical properties test}

Test the impact and compression mechanical properties of the prepared samples. The comparison test between normal temperature $\left(20^{\circ} \mathrm{C}\right)$ and low temperature $\left(-40^{\circ} \mathrm{C}\right)$ is carried out.The impact test samples are $60.0 \mathrm{~mm}$ tubular propellants. Use simply supported beam impact testing machine for testing. The compression test samples are tubular propellant particles with an aspect ratio of 1:1 and tested by the universal material testing machine.

\subsection{Closed bomb test}

The volume of closed bomb is $102.85 \mathrm{~cm}^{3}$, the packing density is $0.20 \mathrm{~g} \cdot \mathrm{cm}^{-3}$ and the ignition pressure is $10.0 \mathrm{MPa}$.The $\mathrm{p}$ - $\mathrm{t}$ curves of nitroguanidine propellants are obtained through the test and then the u-p curves are obtained after data processing.

\section{Results and discussion}

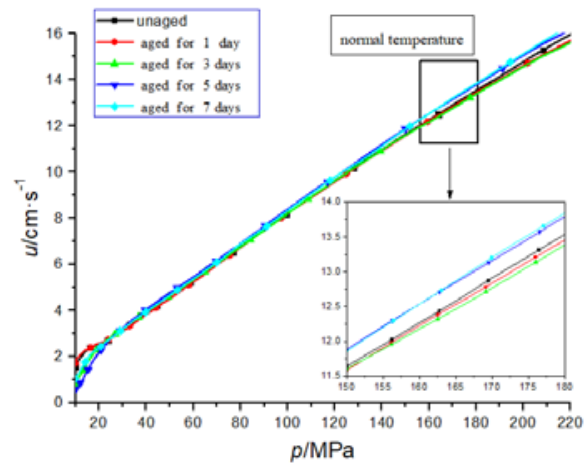

(1) u-p curve at normal temperature

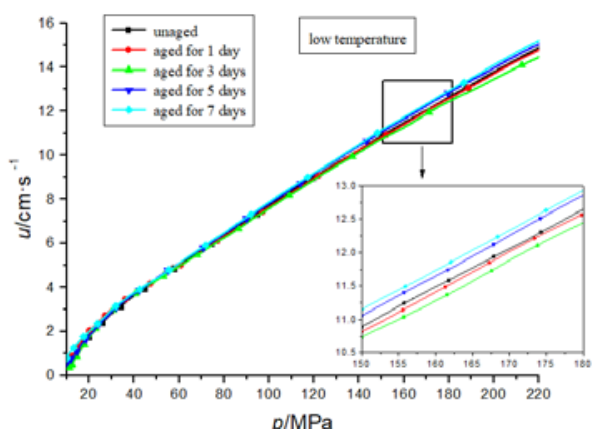

(2)u-p curve at low temperature

Fig. 2. Constant volume burning u-p curves of propellants with different aging times.

Fig. 2 is constant volume combustion u-p curves of propellants with different aging times at low and normal temperature.It can be seen from the partial enlargement of $u-p$ curves, the basic trend of burning rate under normal temperature conditions is: aged for 7 days >aged for 5 days > unaged >aged for 1 day >aged for 3 days; Under low temperature conditions, the burning rate of propellant samples has the same law as normal temperature.

Use unaged propellant as a reference batch, calculate the relative steepness of the constant volume combustion test of the propellant with different aging times at low and normal temperature, as shown in Table 1. Only the propellant samples aged for 3 days have a relative steepness that less than 1 day at low and normal temperature.

Table 1. Relative steepness of propellants with different aging times.

\begin{tabular}{ccc}
\hline \multirow{2}{*}{ Aging time } & \multicolumn{2}{c}{ Relative steepness } \\
& Normal temperature & Low temperature \\
\hline unaged & 1 & 1 \\
1 day & 0.9692 & 1.0042 \\
3 days & 0.9886 & 0.9958 \\
5 days & 1.0064 & 1.0179 \\
7 days & 1.0253 & 1.0545 \\
\hline
\end{tabular}


As shown in Table 2, it can be seen from the results of the impact strength test, under normal and low temperature conditions, the impact strength of the propellant samples aged for 5 days is the highest, followed by the samples aged for 3 days.Under low temperature conditions, the impact strength of samples aged for 1 day and 7 days is lower than that of unaged samples.

Table 2. Impact strength of propellants with different aging times.

\begin{tabular}{ccc}
\hline \multirow{2}{*}{ Aging time } & \multicolumn{2}{c}{ Impact strength $\left(\mathrm{kJ} \cdot \mathrm{m}^{-2)}\right.$} \\
& Normal temperature & Low temperature \\
\hline unaged & 10.636 & 6.086 \\
1 day & 10.429 & 4.804 \\
3 days & 12.906 & 6.247 \\
5 days & 14.159 & 6.444 \\
7 days & 11.892 & 5.532 \\
\hline
\end{tabular}

Use the universal material testing machine to test the effect of aging time on the compressive strength of nitroguanidine propellants. The compression speed is $10 \mathrm{~mm} / \mathrm{min}$. The test results are shown in Table 3:

Table 3. Compressive strength of propellants with different aging times.

\begin{tabular}{ccccc}
\hline \multirow{2}{*}{ Aging time } & \multicolumn{2}{c}{ Compressive strength(MPa) } & \multicolumn{2}{c}{ Compression rate(\%) } \\
\cline { 2 - 5 } & $\begin{array}{c}\text { Normal } \\
\text { temperature }\left(20^{\circ} \mathrm{C}\right)\end{array}$ & $\begin{array}{c}\text { Low } \\
\text { temerature }(-40 \\
\left.{ }^{\circ} \mathrm{C}\right)\end{array}$ & $\begin{array}{c}\text { Normal } \\
\text { temperature }\left(20^{\circ} \mathrm{C}\right)\end{array}$ & $\begin{array}{c}\text { Low } \\
\text { temprature }(-40 \\
\left.{ }^{\circ} \mathrm{C}\right)\end{array}$ \\
unaged & 48.73 & 65.37 & 14.35 & 8.36 \\
1 day & 45.79 & 62.30 & 18.42 & 15.96 \\
3 days & 47.53 & 65.97 & 18.71 & 14.24 \\
5 days & 47.44 & 65.88 & 18.11 & 14.19 \\
7 days & 46.59 & 66.40 & 18.43 & 12.87 \\
\hline
\end{tabular}

It can be seen from the table 3 that different aging times have little effect on the compressive strength of propellants and the unaged propellants have the largest compressive strength at normal temperature. The compressive strength of propellants aged for 7 days at low temperature is the highest, which is only $1.57 \%$ higher than that of the unaged propellants. However, as the aging time increases, the compression rate of the propellants becomes larger. This is because with the extension of the aging time, the solvent penetration in the unformed materials will be more complete, and the unplasticized nitrocellulose will be plasticized in this process. Therefore, the toughness of the propellants increases, and the compression rate increases.

The comprehensive mechanical properties test results show that the aging time has an impact on the strength and toughness of the propellants, and the appropriate aging time effectively improves the impact strength of the nitroguanidine propellants at normal temperature.The compression strength of the propellants does not change much, but the compression rate is significantly improved.

\section{Conclusion}

It can be seen from the related experimental results that only the proper aging time can improve the combustion performance and mechanical properties of the propellants. 
Among the five samples, only propellants aged for 3 days have a relative steepness of less than 1 at low and normal temperature;Propellants aged for 5 days have the highest impact resistance at low temperature, followed by the propellants aged for 3 days;Propellants aged for 7 days have the highest compressive strength at low temperature, followed by the propellants aged for 3 days;Propellants aged for 1 day have the highest compression rate at low temperature, followed by the propellants aged for 3 days.

Combining the combustion performance and mechanical properties, the aging time of 3 days is the more suitable time in the aging process.

\section{Reference}

1. [1] Zhang F, Liu Z, Du P, et al. Thermal Decomposition Kinetics of Nitroguanidine Propellant under Different Pressures[J]. Propellants, Explosives, Pyrotechnics, 2018, 43(4): 390-397.

2. [2] Liu Z, Zhang F, Du P, et al. Effect of NQ content on the thermal decomposition of nitroguanidine propellant using isoconversional methods[J]. Journal of Thermal Analysis and Calorimetry, 2019, 137(2): 473-480.

3. [3] Zhang Lina, Nan Fengqiang, Shen Wanwu. Research on the Flame Retardant Effect of Super-porous Nitroguanidine Propellant Coated with Different Coating Contents of $\mathrm{TiO}<\mathrm{SUB}>2</ \mathrm{SUB}>$. 2018, 436(1)

4. [4] Binbin Wang, Xin Liao, Zeshan Wang, et al. Effects of particle size and morphology of NQ on thermal and combustion properties of triple-base propellants. 2018, 193:123-132.

5. [5] Wan Wu Shen, Feng Qiang Nan, Chun Jiu Zhu, et al. Effects of Formulation and Temperature on the Rheological Behavior of Nitroguanidine-Based Propellants. 2018, 4564:262-268.

6. [6] Le Qi, Zhongliang Ma, Jiahao Liang, et al. Thermomechanical investigation on the effect of nitroguanidine on the thermal expansion coefficient and glass transition temperature of double-base gun propellant. 2019, 8(5):4264-4272.

7. [7] Etienne Comtois, Basil D. Favis, Charles Dubois. Phase transitions and mechanical properties of nitrocellulose plasticized by glycidyl azide polymer and nitroglycerine. 2020, 60(9):2301-2313.

8. [8] Li Gong, Jianmin Li, Yuping Li, et al. Combustion Properties of Composite Propellants Based on Two Kinds of Polyether Binders and Different Oxidizers. 2020, 45(10):1634-1644. 